\title{
Quantum Simulation of the Quantum Rabi Model in a Trapped Ion
}

\author{
Dingshun Lv, ${ }^{1}$ Shuoming An, ${ }^{1}$ Zhenyu Liu, ${ }^{1}$ Jing-Ning Zhang, ${ }^{1, *}$ Julen S. Pedernales, ${ }^{2,3}$ \\ Lucas Lamata, ${ }^{2}$ Enrique Solano, ${ }^{2,4,5}$ and Kihwan Kim ${ }^{1, \dagger}$ \\ ${ }^{1}$ Center for Quantum Information, Institute for Interdisciplinary Information Sciences, \\ Tsinghua University, Beijing 100084, People's Republic of China \\ ${ }^{2}$ Department of Physical Chemistry, University of the Basque Country UPV/EHU, \\ Apartado 644, 48080 Bilbao, Spain \\ ${ }^{3}$ Institute for Theoretical Physics and IQST, Albert-Einstein-Allee 11, \\ Universität Ulm, D-89069 Ulm, Germany \\ ${ }^{4}$ IKERBASQUE, Basque Foundation for Science, Maria Diaz de Haro 3, 48013 Bilbao, Spain \\ ${ }^{5}$ Department of Physics, Shanghai University, Shanghai 200444, China
}

(Received 21 November 2017; revised manuscript received 6 February 2018; published 30 April 2018)

The quantum Rabi model, involving a two-level system and a bosonic field mode, is arguably the simplest and most fundamental model describing quantum light-matter interactions. Historically, due to the restricted parameter regimes of natural light-matter processes, the richness of this model has been elusive in the lab. Here, we experimentally realize a quantum simulation of the quantum Rabi model in a single trapped ion, where the coupling strength between the simulated light mode and atom can be tuned at will. The versatility of the demonstrated quantum simulator enables us to experimentally explore the quantum Rabi model in detail, including a wide range of otherwise unaccessible phenomena, as those happening in the ultrastrong and deep strong-coupling regimes. In this sense, we are able to adiabatically generate the ground state of the quantum Rabi model in the deep strong-coupling regime, where we are able to detect the nontrivial entanglement between the bosonic field mode and the two-level system. Moreover, we observe the breakdown of the rotating-wave approximation when the coupling strength is increased, and the generation of phonon wave packets that bounce back and forth when the coupling reaches the deep strongcoupling regime. Finally, we also measure the energy spectrum of the quantum Rabi model in the ultrastrong-coupling regime.

DOI: 10.1103/PhysRevX.8.021027

Subject Areas: Atomic and Molecular Physics, Particles and Fields, Quantum Physics

\section{INTRODUCTION}

The interaction between light and matter is one of the most fundamental and ubiquitous physical processes. The semiclassical Rabi model was proposed in 1936 to analyze the effect of a varying, weak magnetic field on an oriented atom possessing nuclear spin [1]. It describes the dipolar interaction between a classical monochromatic field and a two-level system, successfully explaining the challenging experimental data in Ref. [2]. When the field is promoted to a quantum description, resulting in the simplest fully

\footnotetext{
*Corresponding author. jnzhang13@mail.tsinghua.edu.cn

Corresponding author. kimkihwan@mail.tsinghua.edu.cn

Published by the American Physical Society under the terms of the Creative Commons Attribution 4.0 International license. Further distribution of this work must maintain attribution to the author(s) and the published article's title, journal citation, and DOI.
}

quantum model of light-matter interaction, it is called the quantum Rabi model (QRM). Typically, the coupling strength in a light-matter system is much lower than the field frequency. In this scenario, the QRM can be simplified to the Jaynes-Cummings model (JCM) under the rotating-wave approximation (RWA) [3]. The JCM is an analytically solvable model that has been studied in cavity quantum electrodynamics [4-6], atomic physics [7], quantum dots [8], circuit quantum electrodynamics (cQED) $[9,10]$, and trapped ions [11-14], among other quantum platforms. Recent experimental achievements have shown the accessibility to the ultrastrong-coupling (USC) regime [15] or even to the deep strong-coupling (DSC) regime $[16,17]$, where the coupling strength is comparable to or larger than the mode frequency. In these strong-coupling regimes, the RWA breaks down, rendering the JCM as a restricted description of the system, and requiring the use of the full QRM to correctly describe the emerging physical phenomena [18]. It is noteworthy to mention that, in such regimes, exotic dynamical properties of lightmatter interaction [19] and potential applications to 
quantum information technologies [20] have been predicted and proposed.

The Hamiltonian associated with the QRM can be expressed as $(\hbar=1)$

$$
\hat{H}_{\mathrm{QRM}}=\frac{\omega_{0}}{2} \hat{\sigma}_{z}+\omega_{m} \hat{a}^{\dagger} \hat{a}+g\left(\hat{\sigma}_{+}+\hat{\sigma}_{-}\right)\left(\hat{a}+\hat{a}^{\dagger}\right),
$$

where $\hat{a}^{\dagger}(\hat{a})$ is the creation (annihilation) operator in the Fock space of a bosonic mode with frequency $\omega_{m}$, and $\hat{\sigma}_{+}\left(\hat{\sigma}_{-}\right)=|\uparrow\rangle\langle\downarrow|(|\downarrow\rangle\langle\uparrow|)$ and $\hat{\sigma}_{z}=|\uparrow\rangle\langle\uparrow-\mid \downarrow\rangle\langle\downarrow|$ are the raising (lowering) and the Pauli $z$-basis operators, respectively, of a two-level system, an effective spin with energy splitting $\omega_{0}$, and $g$ is the coupling strength. Three major coupling regimes are defined depending on the ratio between the coupling strength $g$ and the field mode frequency $\omega_{m}$, namely, the weak-coupling or JaynesCummings regime, with $g / \omega_{m} \ll 0.1$, the USC regime, with $0.1 \lesssim g / \omega_{m}$, and the DSC regime, with $1 \lesssim g / \omega_{m}$. In the Jaynes-Cummings regime, corotating terms $\hat{a} \hat{\sigma}_{+}$and $\hat{a}^{\dagger} \hat{\sigma}_{-}$dominate in the Hamiltonian Eq. (1). In other words, the RWA is valid and the QRM reduces to the JCM, of which the whole Hilbert space consists of a series of independent twofold subspaces $\{|\uparrow, n\rangle,|\downarrow, n+1\rangle\}$, where $n$ is the quantum number of Fock state $|n\rangle$. In the USC or DSC regimes, however, the counterrotating terms $\hat{a} \hat{\sigma}_{-}$and $\hat{a}^{\dagger} \hat{\sigma}_{+}$cannot be neglected. These two terms connect different twofold subspaces, break the simple structure of the JCM, and do not preserve the number of excitations. These excitation-nonconserving terms make it difficult to find an analytical solution of the QRM, which was found only recently [21]. Although the total excitation number is not conserved, it has been pointed out that the parity operator $\hat{\Pi}=\sigma_{z} e^{-i \pi \hat{a}^{\dagger} \hat{a}}$, with eigenvalues \pm 1 , is still a conserved quantity. The Fock space can be divided in two parts, each spanned by states of a given parity. The notion of parity chain was introduced in Ref. [19] to address the set of states $|\downarrow(\uparrow), n\rangle$ with the same parity ordered in ascending $n$. It is known that wave packets in the Fock space evolve within the same parity chains when in the USC or DSC regimes [19]. Recently, it has also been predicted that the ground states of the QRM in the USC or DSC regimes reveal the conservation of parity and the entanglement between spin and mode with large excitation numbers [22-25].

In recent years, experimental efforts have been made to reach the DSC regime [16,17], which stimulates the study of exotic physics of the QRM in the USC or DSC regimes. The phenomenon of photon number wave packets bouncing back and forth along the parity chains [19] has been observed in a classical simulator of a photonic waveguide system [26] and in analog and digital quantum simulations in cQED systems [27,28]. However, the study of the ground state in DSC regime is still an open challenge [22].
In this work, we report the analog quantum simulation of the quantum Rabi model with a single trapped ion for all relevant coupling regimes. Among the results, we generate and observe the ground state of the QRM in the DSC regime in a trapped-ion quantum simulator for the first time. We demonstrate the full controllability and tunability of the QRM in a single trapped-ion system as proposed in Ref. [22], which enable us to generate the exotic ground state in the DSC regime by the adiabatic transfer from the simple ground state of the JCM. Moreover, we apply the capability of the ground-state preparation to experimentally measure the energy spectrum of the QRM Hamiltonian Eq. (1).

\section{TRAPPED-ION SYSTEM}

In our experiment, a radio frequency Paul trap is used to spatially confine an ${ }^{171} \mathrm{Yb}^{+}$ion that is then cooled down to its motional ground state by standard sideband cooling techniques [12] after Doppler cooling. In the low-energy regime, the motion of the ion can be well approximated to that of a harmonic oscillator, and two energy levels of the hyperfine manifold of its electronic ground state can be used as a qubit. In particular, we encode the two-level system in the levels $\left|F=1, m_{F}=0\right\rangle \equiv|\uparrow\rangle$ and $\left|F=0, m_{F}=0\right\rangle \equiv|\downarrow\rangle$ of the $S_{1 / 2}$ hyperfine manifold, which have a transition frequency $\omega_{\mathrm{hf}}=(2 \pi) 12.642812 \mathrm{GHz}$. We employ a radial vibrational mode of frequency $\omega_{X}=(2 \pi) 2.498 \mathrm{MHz}$ as the bosonic degree of freedom of our simulator. The total Hamiltonian of the system is composed of the uncoupled Hamiltonian and the ion-laser interaction. The uncoupled Hamiltonian describing such a system is given by $\hat{H}_{0}=\left(\omega_{\mathrm{hf}} / 2\right) \hat{\sigma}_{z}+\omega_{X} \hat{a}^{\dagger} \hat{a}$. When a pair of counterpropagating Raman laser beams is driven onto the ion, the ion-laser interaction is described by

$$
\hat{H}_{\text {ion-laser }}=\hbar \Omega_{b, r} \cos \left(k_{b, r} \hat{x}-\omega_{b, r} t+\phi_{b, r}\right) \hat{\sigma}_{x} .
$$

Here, $\Omega_{b, r}$ is the Rabi strength proportional to the product of both laser field amplitudes for blue-sideband or redsideband transitions, $\Delta k_{b, r}$ is the net wave vector component of the Raman laser beams on the direction of the motion of the ion, $\hat{x}=x_{0}\left(\hat{a}+\hat{a}^{\dagger}\right)$ is the position operator of the ion, with ground-state wave packet width $x_{0}=\sqrt{\hbar / 2 M \omega_{X}}$, where $M$ is the mass of the ${ }^{171} \mathrm{Yb}^{+}$ ion, and $\omega_{b, r}$ and $\phi_{b, r}$ are the differences of frequencies and phases of the Raman laser beams, respectively [14].

Under suitable optical and vibronic RWAs, and also in the Lamb-Dicke regime, the ion-laser interaction can be turned into an (anti-)Jaynes-Cummings interaction by tuning the laser frequency close to the red (blue) sideband as $\omega_{r}=\omega_{\mathrm{hf}}-\omega_{X}-\delta_{r} \quad\left(\omega_{b}=\omega_{\mathrm{hf}}+\omega_{X}-\delta_{b}\right)$, with a small detuning $\delta_{r(b)} \ll \omega_{X}$ in the most general case. Red-sideband and blue-sideband interactions are described in the interaction picture with respect to the uncoupled 
Hamiltonian $\hat{H}_{0}=\left(\omega_{\mathrm{hf}} / 2\right) \hat{\sigma}_{z}+\omega_{X} \hat{a}^{\dagger} \hat{a}$ by the Hamiltonians $[14,29]$

$$
\begin{aligned}
\hat{H}_{\text {red }}(t) & =\frac{\eta \Omega_{r}}{2}\left(\hat{a} \hat{\sigma}_{+} e^{i \delta_{r} t}+\hat{a}^{\dagger} \hat{\sigma}_{-} e^{-i \delta_{r} t}\right), \\
\hat{H}_{\text {blue }}(t) & =\frac{\eta \Omega_{b}}{2}\left(\hat{a}^{\dagger} \hat{\sigma}_{+} e^{i \delta_{b} t}+\hat{a} \hat{\sigma}_{-} e^{-i \delta_{b} t}\right) .
\end{aligned}
$$

Here, $\eta=\Delta k_{b, r} x_{0}$ is the Lamb-Dicke parameter.

When both red- and blue-sideband interactions are simultaneously applied with equal strength, such that $\Omega=\Omega_{r}=\Omega_{b}$, one can write the total Hamiltonian in the interaction picture as

$$
\hat{H}_{b r}(t)=\frac{\eta \Omega}{2} \hat{\sigma}_{+}\left(\hat{a} e^{i \delta_{r} t}+\hat{a}^{\dagger} e^{i \delta_{b} t}\right)+\text { H.c. }
$$

Equation (4) corresponds to the interaction picture Hamiltonian with respect to the uncoupled Hamiltonian $\hat{H}_{0}^{\prime}=\left(\delta_{b}+\delta_{r}\right) / 4 \hat{\sigma}_{z}+\left(\delta_{b}-\delta_{r}\right) / 2 \hat{a}^{\dagger} \hat{a}$ for the following effective Hamiltonian,

$$
\begin{aligned}
\hat{H}_{\mathrm{eff}}= & \frac{\left(\delta_{b}+\delta_{r}\right)}{4} \hat{\sigma}_{z}+\frac{\left(\delta_{b}-\delta_{r}\right)}{2} \hat{a}^{\dagger} \hat{a} \\
& +\frac{\eta \Omega}{2}\left(\hat{\sigma}_{+}+\hat{\sigma}_{-}\right)\left(\hat{a}+\hat{a}^{\dagger}\right),
\end{aligned}
$$

where the parameters of the simulated QRM can be associated with the experimental ones as $\omega_{0}=\left(\delta_{b}+\delta_{r}\right) / 2$, $\omega_{m}=\left(\delta_{b}-\delta_{r}\right) / 2$, and $g=(\eta \Omega / 2)$. Thus, such an experimental setup serves as a quantum simulator of the QRM, where one can simulate a wide range of coupling regimes by suitably tuning the laser intensities and detunings to match the desired ratio $g / \omega_{m}$. It is important to point out that the observables of interest $\left\{\hat{a}^{\dagger} \hat{a}, \hat{\sigma}_{z},|n\rangle\langle n|\right\}$ commute with all the adopted interaction-picture transformations, which are always with respect to a Hamiltonian of the form $\alpha \hat{a}^{\dagger} \hat{a}+\beta \hat{\sigma}_{z}$, such that their expectation values will remain unaltered in the laboratory reference frame [22].

\section{COUPLING REGIMES AND BREAKDOWN OF THE RWA}

For the experiment, we fix the coupling strength to $g=(2 \pi) 12.5 \mathrm{kHz}$, and the detuning of the red sideband to $\delta_{r}=0$, leaving $\delta_{b}$ as a tunable parameter. In this manner we will be simulating a resonant QRM where the ratio $g / \omega_{m}$ will be determined by the selected detuning $\delta_{b}$. We experimentally explore three paradigmatic coupling regimes, namely, the Jaynes-Cummings, the USC, and the DSC regimes, accordingly selecting the values of the detuning for the blue sideband as $\delta_{b}=2 \omega_{m}=$ $(2 \pi)\{625,83.4,41.6\} \mathrm{kHz}$, which correspond to the ratios $g / \omega_{m}=\{0.04,0.6,1.2\}$, respectively.

The experiment is carried out as follows. First, we perform standard Doppler and sideband cooling, which prepares the system in the state $|\downarrow, n=0\rangle$ [30], and then we transfer the system to the initial state $|\uparrow, n=0\rangle$ by applying a carrier $\pi$ pulse. After that, we turn on the redsideband and blue-sideband transitions, with suitably chosen intensities and detunings, to implement the QRM Hamiltonian in the desired regime. We observe the dynamics of the QRM by measuring the average excitations of the spin $\left\langle\hat{\sigma}_{+} \hat{\sigma}_{-}\right\rangle$and the phononic degrees of freedom $\left\langle\hat{a}^{\dagger} \hat{a}\right\rangle$ at specific evolution times $t$. We measure the average excitation of the spin $\left\langle\hat{\sigma}_{+} \hat{\sigma}_{-}\right\rangle$by spin-dependent fluorescence detection, where only the spin- $|\uparrow\rangle$ state scatters photons. We obtain the average excitation of phonons $\left\langle\hat{a}^{\dagger} \hat{a}\right\rangle$ from the measured phonon-number distribution, which is deduced from fitting the blue-sideband signal to Eq. (C1) in Appendix C.

In Figs. 1(a) and 1(d), the measurements for the simulation of the Jaynes-Cummings regime are plotted. Rabi oscillations, with a complete collapse and posterior revival of the excitation probability of the two-level system, are clearly observed. In the same manner, the average number of phonons in the bosonic mode oscillates between 0 and 1 , consistent with the notion that the wave function of the system should live in the space spanned by the corresponding JCM doublet $\{|0, \uparrow\rangle,|1, \downarrow\rangle\}$, as expected for such a regime. Figures 1 (b) and 1(e) show the evolution of the same initial state in the USC regime for the coupling ratio $g / \omega_{m}=0.6$. In this case, collapses and revivals of the excitation probability are not complete and the average number of phonons exceeds 1 , indicating that the dynamics no longer happen exclusively in the JCM doublet. This departure from the JCM physics is associated with the breakdown of the RWA due to the large coupling ratio. In the DSC regime, plotted in Figs. 1(c) and 1(f) for the coupling ratio $g / \omega_{m}=1.2$, the effects of the RWA breakdown are even clearer, where not even oscillations can be identified and where the average number of phonons grows above 6 for the plotted example. We also show in Fig. $1(\mathrm{~g})$ the evolution of the total excitation number $\langle\hat{N}\rangle=\langle\mid \uparrow\rangle\langle\uparrow \mid\rangle+\langle\hat{n}\rangle$, which is a conserved quantity when the RWA is valid, but has a dynamical behavior as soon as the RWA breaks down.

\section{DSC REGIME AND PHONON WAVE PACKETS}

We focus now on the DSC regime and explore two scenarios, namely, the degenerate case with $\omega_{0}=0$ and the nondegenerate case with $\omega_{0} \neq 0$. In contrast to the weakcoupling regime (JCM regime) of the natural light-matter interaction, in the USC or DSC regime the spin-field interaction can survive even when the spin is degenerate. Therefore, we expect to observe quantum dynamics due to the strong-coupling effect. We note that the degenerate case has been investigated thoroughly for the production of Schrödinger's cat states [31-36] and the calibration of two-qubit Mølmer-Sørensen gates [37-42] in trapped-ion 

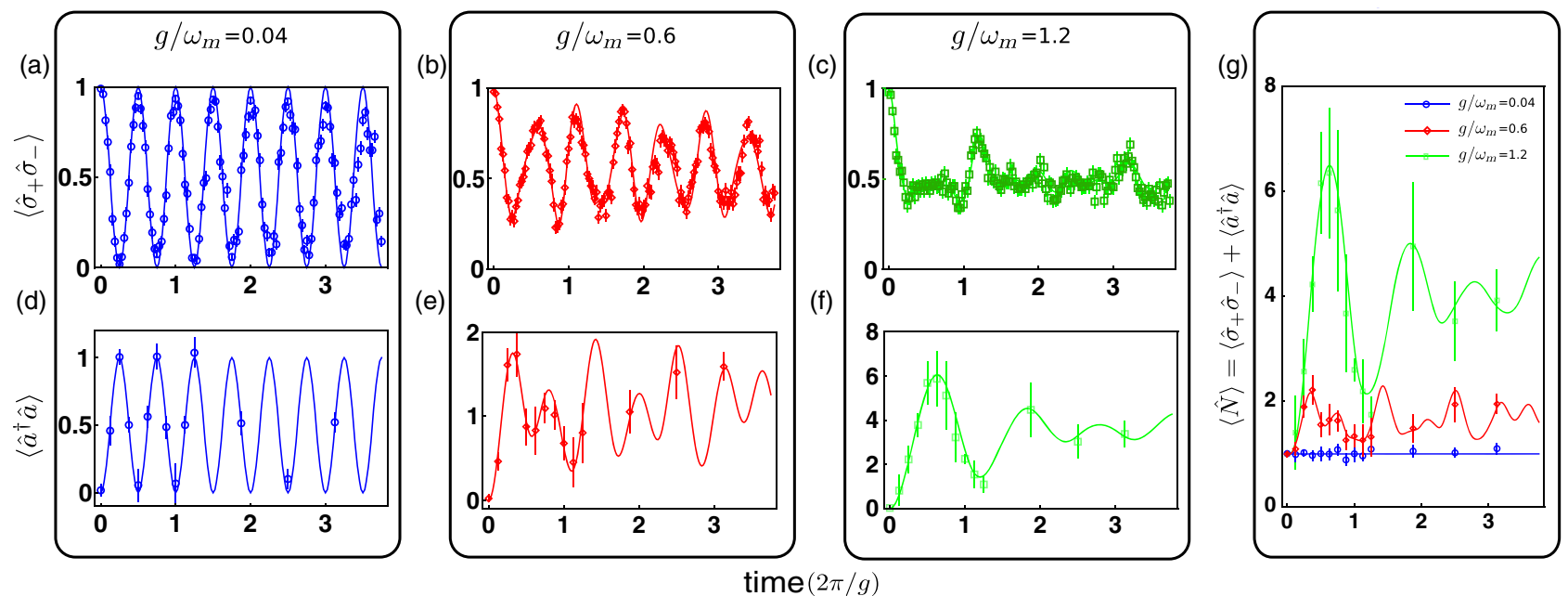

FIG. 1. Spin and phonon dynamics under the QRM for different coupling regimes. Panels (a)-(c) correspond to the population of the excited state of the two-level system for the coupling ratios $g / \omega_{m}=0.04,0.6$, and 1.2 , respectively. Panels (d)-(f) correspond to the evolution of the average number of phonons for the same coupling ratios. Finally, panel $(\mathrm{g})$ shows the evolution of the total number of excitations for the three cases considered above. In all panels, theoretical predictions are plotted with continuous lines, while dots and their associated error bars represent the experimental data. The lower number of points in the plots of $\left\langle a^{\dagger} a\right\rangle$ is because their measurement process (described in Appendix C) is more time-consuming.

systems. Although the spin-dependent force is a welldeveloped technique for the trapped-ion community, it has never been viewed as the simulation of a special case of the QRM. For this experiment, we fix the coupling strength to $g=(2 \pi) 12.5 \mathrm{kHz}$, and vary $\delta_{r}$ and $\delta_{b}$, while keeping always a ratio $g / \omega_{m}=1.25$. For the degenerate case, we use detunings $\delta_{b}=-\delta_{r}=(2 \pi) 10 \mathrm{kHz}$, while for the nondegenerate case, we use $\delta_{r}=0$ and $\delta_{b}=(2 \pi) 20 \mathrm{kHz}$, which corresponds to $\omega_{0}=0.8 g$. For the initial state, we choose the ground state of the JC model $|\downarrow, 0\rangle$, which should have no dynamical properties when the coupling strength $g / \omega_{m}$ is small enough. In Fig. 2, we show that the situation is different when considering the DSC regime. First of all, in Figs. 2(a) and 2(b), we show the evolution of (a)

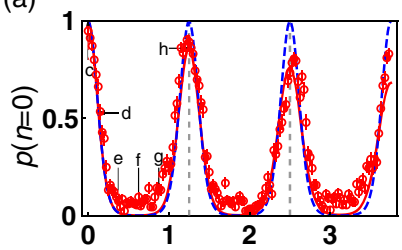

(b)

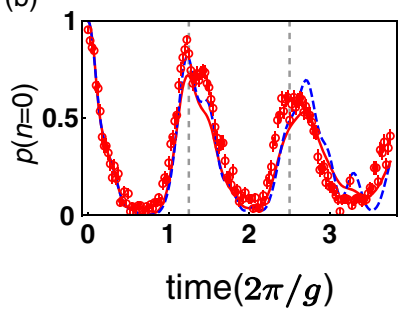

(c)

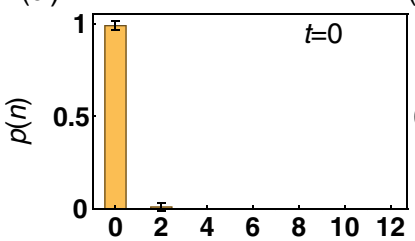

(f)

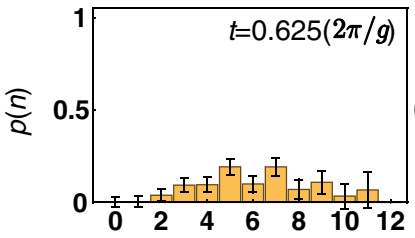

(d)

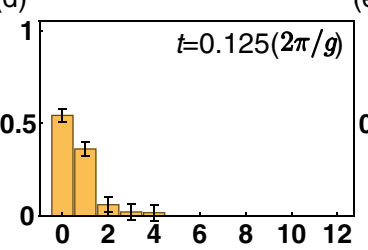

(g)

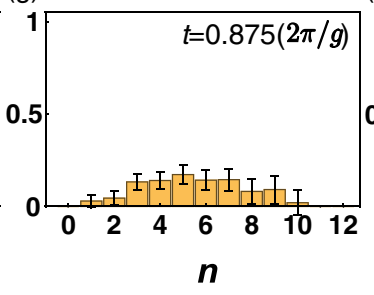

(e)

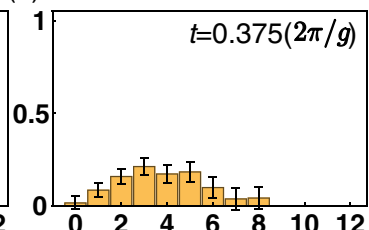

(h)

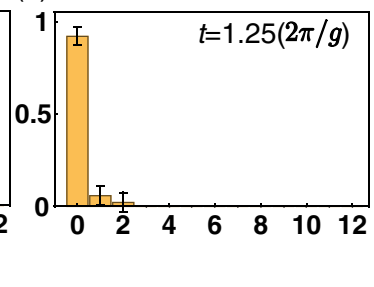

FIG. 2. Phonon-number wave packets bouncing back and forth in the DSC regime. In panels (a) and (b) we plot the population of state $|n=0\rangle$, after tracing out the spin, as the system evolves under the QRM in the DSC regime. In particular, panel (a) shows the degenerate case, $\omega_{0}=0$, and (b) the nondegenerate case, with $\omega_{0}=0.8 \mathrm{~g}$. For both cases, the coupling ratio is fixed to $\mathrm{g} / \omega_{m}=1.25$. Dashed and solid lines represent theoretical calculations with and without decoherence of the motional mode, respectively. The vertical dashed lines indicate the motional revival times $k\left(2 \pi / \omega_{m}\right)$, where $k=1,2, \ldots$ The data points with error bars correspond to the experimental results. We obtain the zero-phonon population following the method in Ref. [14] after tracing out the spin. Panels (c)-(h) show the phononnumber distribution sampled at several instants during one period $T=\left(2 \pi / \omega_{m}\right)=100 \mu$ s of the QRM Hamiltonian for the degenerate case. The phonon distribution is obtained by fitting the standard blue-sideband signals to Eq. (C1) after tracing out the spin (see Appendix C). At the first revival time, the phonon state is back to the initial state as predicated by the QRM. The imperfections can be attributed to decoherence of the motional degrees of freedom. 
the population of Fock state $|0\rangle$, after tracing out the spin degree of freedom. For the degenerate case, we can clearly observe that this population collapses to zero and that it is stabilized at zero except every one period of the mode frequency, $k\left(2 \pi / \omega_{m}\right), k$ being an integer, where a full revival of the population is detected. On the other hand, the nondegenerate case shows long-time degradation of these revivals, as it was predicted in Ref. [19].

Additionally, we sample several points during one period $T=\left(2 \pi / \omega_{m}\right)=100 \mu$ s of the evolution of the degenerate case and measure its phonon distribution, as shown in Figs. 2(c)-2(h). At time zero, the population is concentrated on Fock state $|0\rangle$, and as time elapses higher Fock states are populated. In fact, the quantum dynamics is exactly solvable and the time-dependent state evolves as $(1 / \sqrt{2})(|\downarrow, \alpha(t)\rangle+|\uparrow,-\alpha(t)\rangle)$, with $\alpha(t)=$ $\left(g / 2 \omega_{m}\right)\left(1-e^{i 2 \omega_{m} t}\right)$ [34]. The evolution resembles a wave packet that travels along a chain of Fock states up to a maximum determined by $\sim 4\left(g / \omega_{m}\right)^{2}$ and then comes back to the initial states at one period of the mode frequency. This phenomenon was theoretically predicted in Ref. [19] as characteristic of the DSC regime, and it is referred to as the bouncing back and forth of phonon-number wave packets. We note that similar reconstruction of the state can be found in Ref. [34] in the context of spin-dependent force. It has also been pointed out that the simulation of the Dirac equation can be interpreted as the simulation of the QRM in the DSC regime with infinite ratio by setting $\omega_{m}=0[22,43-45]$.

\section{ADIABATIC GROUND-STATE PREPARATION}

As mentioned in the previous section, the ground state of the QRM in the Jaynes-Cummings regime $\left(g \ll \omega_{m}\right)$ is given by the state $|\downarrow, 0\rangle$, while the ground state of the QRM in the DSC regime is a nontrivial state where spin and field are entangled, and which, to the best of our knowledge, has never been implemented in a physical quantum platform.

In our experiment, we generate the ground state of the QRM in the DSC regime by starting in the ground state of the Jaynes-Cummings regime, the state $|\downarrow, 0\rangle$, and adiabatically increasing the coupling ratio $g / \omega_{m}$ towards the DSC regime. To achieve this, one could choose to either increase $g=\eta \Omega / 2$, which can be done by raising the laser intensity, or decrease $\omega_{m}=\left(\delta_{b}-\delta_{r}\right) / 2$. Because it is experimentally more feasible to manipulate the detuning of the Raman lasers than their power, we choose the latter in our experiment. For that, we fix the coupling strength $g$ to be $(2 \pi) 12.5 \mathrm{kHz}$ and $\delta_{r}=0$, leaving $\delta_{b}$ as the only tunable parameter, which we manipulate with an exponential time dependence of the form $\delta_{b}(t)=\left(\delta_{\max }-\delta_{\operatorname{tar}}\right) e^{-t / \tau}+\delta_{\operatorname{tar}}$. Here, we set $\delta_{\max }=(2 \pi) 0.2 \mathrm{MHz}$, while $\delta_{\text {tar }}$ is determined by the ratio $g / \omega_{m}$ we want to reach, and $\tau=\left(t_{\text {tar }} / 10\right)$, $t_{\mathrm{tar}}=300 \mu \mathrm{s}$ being the total duration of the adiabatic process. The adiabaticity of our scheme is guaranteed by the numerical computation of the fidelity between the instantaneous ground state of the Hamiltonian and the adiabatically evolved state, which is shown in Fig. 3(a). Here, the fidelity is defined as $\operatorname{Tr} \sqrt{\sqrt{\rho_{\text {id }}(t)} \rho(t) \sqrt{\rho_{\text {id }}(t)}}$ [46], where $\rho_{\text {id }}$ is the ideal instantaneous ground state obtained from direct diagonalization, and $\rho$ is the state resulting from the numerical calculation of the time evolution. We note that the fidelity of the $|\downarrow, 0\rangle$ state to the ideal ground state in the case of $g / \omega_{m}=0.125$ is $99.8 \%$. With sideband cooling, we are able to prepare the $|\downarrow, 0\rangle$ state with a fidelity of around $99.7 \%$. Therefore, the total infidelity of our prepared ground state with respect to the ideal one is around $0.5 \%$, which is much smaller than the detection capability in our system.

In Fig. 3(b), we show the spin evolution during the adiabatic process for the time interval $\left(0-t_{\mathrm{tar}}\right)$. The plot corresponds to the case $g / \omega_{m}=1.2$, with the cases for other ratios showing similar behavior. At time $t_{\mathrm{tar}}$, the system is expected to be in the ground state of the QRM for the selected coupling regime. In Figs. 3(d)-3(f), we plot the outcome of the phonon distributions correlated with the spin at $t_{\text {tar }}$ for coupling ratios $g / \omega_{m}=1.2,1.5$, and 2.0, respectively (see Appendix C). To measure the phononnumber distribution correlated with state $|\downarrow\rangle$ of the spin, we first perform a spin-dependent fluorescence detection and select the case of no fluorescence, which corresponds to the spin being projected to the $|\downarrow\rangle$ state. Then we drive the blue-sideband transition, monitor the population of the $|\uparrow\rangle$ state, and fit the signal to the function in Eq. (C1) as described in Appendix C. This provides the conditional phonon-number distribution normalized to the probability of being in the spin state $|\downarrow\rangle$. To measure the phononnumber distribution correlated with the spin state $|\uparrow\rangle$, we first flip the spin and then follow the same procedure as that for the case of state $|\downarrow\rangle$.

From Figs. 3(d)-3(f), we clearly observe that larger phonon-number states are populated in the prepared ground states as the ratio $g / \omega_{m}$ increases. We also observe that the populations are distributed mostly along the same parity chain, which is expected from the $Z_{2}$ symmetry of the QRM [19]. We quantify the parity conservation by measuring the expectation value of the parity operator $\hat{\Pi}=\sigma_{z} e^{-i \pi \hat{a}^{\dagger} \hat{a}}$, which has eigenvalues \pm 1 . The measured parities for the states of Figs. 3(d)-3(f) are 0.74(0.08), 0.70 (0.08), and 0.52(0.13), respectively, showing that the prepared ground states in the DSC regime mostly dwell in the same parity chain. As the coupling ratio increases, the measured parity deviates from the ideal parity value +1 due to imperfections in the adiabatic process and the motional heating arising from the occupation of large Fock states.

To verify the quantum coherence maintained within the preparation of the ground state, we reverse the adiabatic process in an attempt to recover the initial ground state $|\downarrow, 0\rangle$. In Fig. 3(b), we can observe how the spin returns to 
(a)

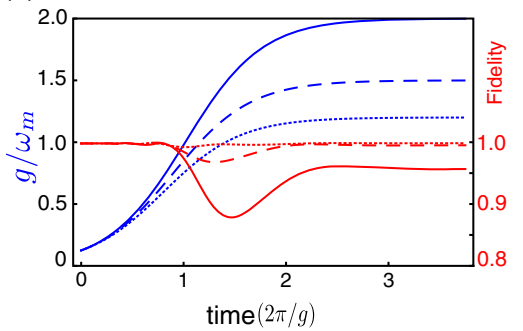

(d)

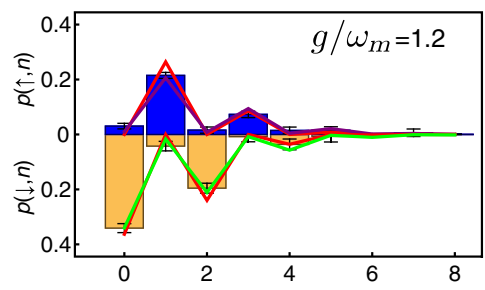

(b)

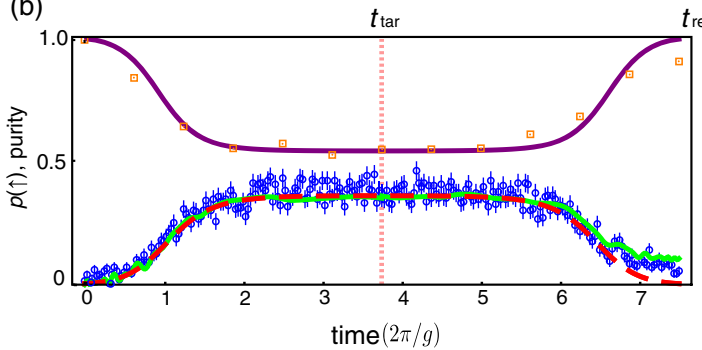

(f)

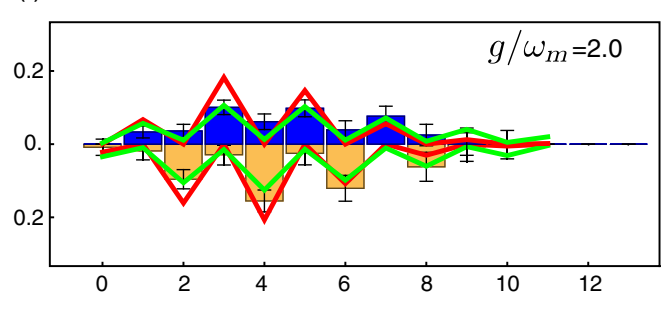

FIG. 3. Adiabatic ground-state preparation of the QRM in the DSC regime. In (a), we show the adiabatic scheme for the preparation of the ground state of the QRM at the DSC ratios $g / \omega_{m}=1.2,1.5$, and 2.0, as starting from the initial JC ratio $g / \omega_{m}=0.125$. The fidelities between the instantaneous ground state and the numerically evolved state quantify the adiabaticity of our process. In (b), we show the evolution of the excitation probability and the purity of spin state during the adiabatic ground-state preparation $\left(0 \leq t \leq t_{\text {tar }}\right)$ and during the reverse process $\left(t_{\mathrm{tar}} \leq t \leq t_{\mathrm{rev}}\right)$. The plot corresponds to the preparation of the ground state at the ratio $g / \omega_{m}=1.2$. The red dashed line and the green solid line are obtained by direct diagonalization of the QRM Hamiltonian and numerical simulation of the adiabatic process, respectively, including heating and dephasing of the motional mode, which are expected experimental imperfections. The blue circles with error bars correspond to the experimental results. The purple line represents the numerically computed purity of the spin, defined as $\operatorname{Tr}\left(\hat{\rho}_{\text {spin }}^{2}\right)$, where $\hat{\rho}_{\text {spin }}$ is the reduced density matrix of the spin after tracing out the motional degree of freedom. The orange squares are the corresponding experimental results, computed from the spin tomography [46,47]. Panels (d)-(f) show the phononnumber distributions correlated with $|\downarrow\rangle$ (lower panel) and $|\uparrow\rangle$ (upper panel), which are obtained by fitting the standard blue-sideband signals after the spin-projective measurement for $g / \omega_{m}=1.2,1.5$, and 2.0, respectively. The red solid line and the green solid line are obtained by direct diagonalization of the QRM Hamiltonian and numerical simulation of the adiabatic process, respectively, including heating and dephasing of the motional mode, which are expected experimental imperfections. Finally, panel (c) shows the purity of the spin and estimated lower bounds for the purity of the whole system, for each tested coupling ratio. The purity of the spin is obtained from the measured reduced density matrix, which was done by spin tomography. The lower bounds of the purities of the whole system are estimated by measuring the probability of being in $|\downarrow, 0\rangle$ after the reverse process at $t_{\mathrm{rev}}$. Note that we get the numerical simulation results in (b)-(f) by solving the Lindblad master equation (see Appendix B).

state $|\downarrow\rangle$. As a complementary proof, we plot the purity of the spin state. To this aim, we trace out the phononic degrees of freedom and measure the density matrix $\hat{\rho}_{\text {spin }}$ associated with the spin degree of freedom [46], from which we calculate the purity, defined as $\operatorname{Tr}\left(\hat{\rho}_{\text {spin }}^{2}\right)$, during the whole process. The degradation of the purity during the preparation of the ground state of the QRM in the DSC regime confirms that the adiabatically prepared ground state is indeed an entangled state, and the subsequent revival of the purity when the adiabatic process is inverted proves that we are able to recover the initial state and therefore that the whole process preserves quantum coherence.

By measuring the probability of recovering the initial state $|\downarrow, 0\rangle$ after the ground-state preparation and reverse process at time $t_{\text {rev }}$, we estimate a lower bound of the purity of the prepared ground state. The revival probabilities are $0.89(0.024), 0.87(0.027)$, and $0.79(0.03)$ for the three ratios $g / \omega_{m}=1.2,1.5$, and 2.0 , which give the lower bounds
0.79(0.042), 0.75(0.047), and 0.62(0.047), respectively. As shown in Fig. 3(c), the reduced-spin purities, taking values 0.545(0.006), 0.514(0.003), and 0.505(0.002), are significantly smaller than the lower bounds of the total system, which prove the existence of entanglement within the prepared ground state at $t_{\mathrm{tar}}$ (See Appendix D).

\section{SPECTRUM}

The ground-state preparation can be extended to study the low-lying energy spectrum of the QRM by coherent spectroscopy [48]. In particular, we have measured the energy spectrum in the region $g / \omega_{m} \in[0,1]$. A $Z_{2}$ parity exists in the QRM model, which divides the Hilbert space in two, namely, a subspace of parity +1 and another of parity -1 [19]. Here, we focus on the energy splittings between the ground state and the first three excited states of opposite parity to the ground state [19]. For that, we have used a relatively weak modulated field as a probe on top of the simulation of the QRM, with the system initially in the 

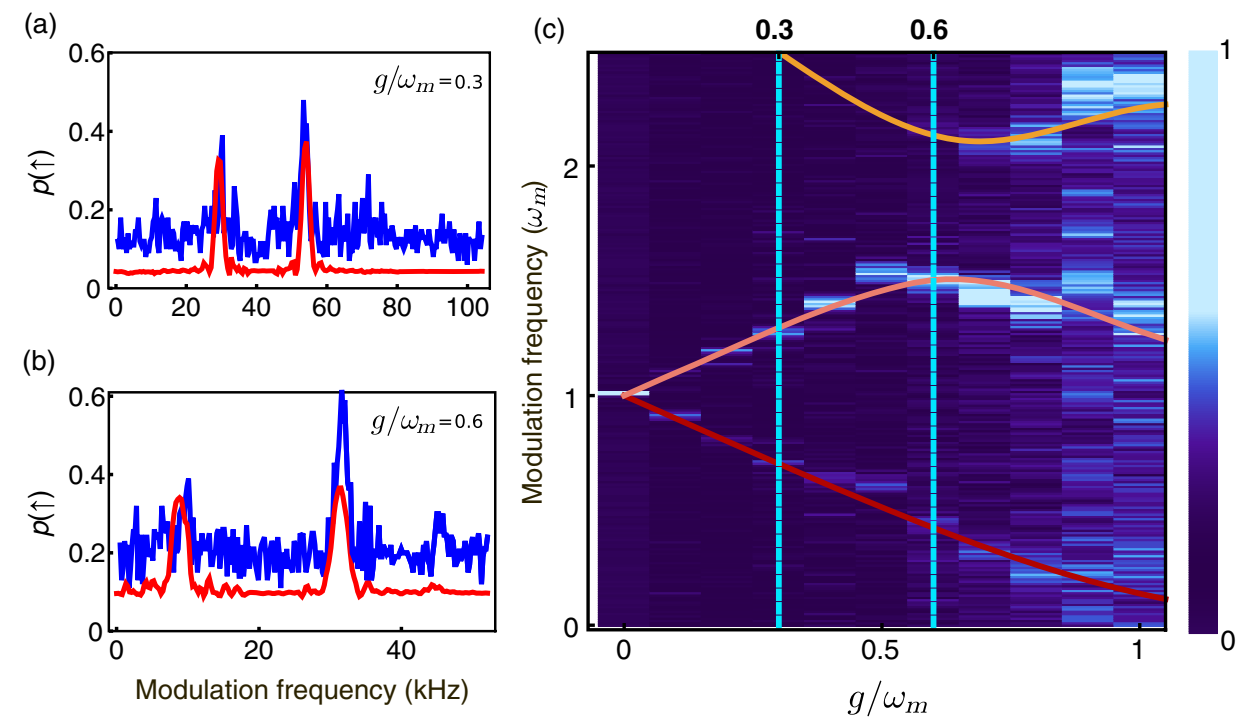

FIG. 4. Spectrum of the QRM. Panels (a) and (b) show, respectively for the regimes $g / \omega_{m}=0.3$ and 0.6 , the population of the excited state of the spin as a function of the modulation frequency of the probe driving. The red curve corresponds to numerical simulation results, while the blue curve shows the experimental data. Panel (c) shows the energy spectrum with the modulation frequency of the probe drive in the vertical axis rescaled by $\omega_{m}$. Note that the energy of the ground state (not plotted) is taken to be zero for all cases. The three continuous curves on top of the plot show the numerically computed energy spectrum of the states with parity opposite to the ground state.

ground state of the corresponding regime. We sweep the frequency of the probe pulse until we detect a transition, and we associate the frequency of the probe to the energy difference of the transition. To generate transitions between states of opposite parity, we use the probe pulse of the form

$$
\hat{H}_{\mathrm{mod}}=\hat{H}_{\mathrm{QRM}}+g_{p} \sin \left(2 \pi \nu_{p} t\right)\left(\hat{\sigma}_{+}+\hat{\sigma}_{-}\right),
$$

where $g_{p}(\ll g)$ is the strength of the modulation field, and $\nu_{p}$ is swept to find the resonant frequencies. In the region $g / \omega_{m}=0.1-0.3, g_{p} / g$ is 0.02 , while the pulse duration is $350 \mu \mathrm{s}$. For the ratios $g / \omega_{m}=0.4-1.0$, the ratio $g_{p} / g$ is 0.01 , with a pulse duration of $450 \mu \mathrm{s}$. Population transfer is clearly seen when $\nu_{p}$ is resonant with the energy splittings as shown in Fig. 4.

\section{CONCLUSION}

We implement the quantum simulation of all relevant coupling regimes of the QRM in a single trapped ion, obtaining direct evidence of the breakdown of the RWA. In the DSC regime, we observe the phonon-number wave packets bounce back and forth as well as collapses and revivals of the initial state, confirming previous theoretical predictions. The adiabatic preparation of the ground state of the QRM in the DSC regime is produced for the first time in a quantum platform, and its reconstruction has enabled us to demonstrate the entanglement present in its ground state. As a direct application of this adiabatic method, we are able to measure the energy splittings between states of different parity and recreate the energy spectrum of the QRM in the
USC regime. In conclusion, our work presents a detailed experimental exploration of the QRM in a wide range of physical regimes. From a theoretical point of view, our work justifies further research in light-matter coupling regimes that are a priori not found in nature, as we are providing a platform where all these regimes can be physically implemented. Our experimental methods can be directly extended to the study of the phase transition in the QRM [49-51] or to the simulation of the Dicke model [52-54] by considering the presence of more ions.

\section{ACKNOWLEDGMENTS}

We thank Xiao Yuan, Xiongfeng Ma, Hyunchul Nha, Jiyong Park, Jaehak Lee, and M. S. Kim for useful discussions on the entanglement verification of the ground state. This work was supported by the National Key Research and Development Program of China under Grants No. 2016YFA0301900 and No. 2016YFA0301901 and the National Natural Science Foundation of China Grants No. 11374178, No. 11574002, and No. 11504197 , MINECO/FEDER FIS2015-69983-P, Ramón y Cajal Grant No. RYC-2012-11391, and Basque Government IT986-16.

\section{APPENDIX A: CALIBRATION OF THE DETUNING OF THE BLUE AND RED SIDEBAND TRANSITIONS}

For the simulation of the case $g / \omega_{m}=0.04$, we set $\delta_{r}=0$, while $\delta_{b}$ takes a much larger value than its corresponding coupling strength. To achieve that 
configuration, we obtain the resonance frequency of the red-sideband transition with the detuning of the bluesideband transition fixed at $\delta_{b}=(2 \pi) 625 \mathrm{kHz}$. For the USC or DSC regime, the coupling strength is comparable to the effective mode frequency, such that we need to carefully deal with the ac-Stark shift introduced by an offresonant excitation of the carrier transition. We measure the ac-Stark shift with a Ramsey experiment and calibrate the shift in the bichromatic pulse within $1 \mathrm{kHz}$ of accuracy. We further improve the frequency precision within a $0.15-\mathrm{kHz}$ range by setting the same detuning $\delta=$ $(2 \pi) 10 \mathrm{kHz}$ with different signs for each beam, similarly to the scheme in Refs. [41,55].

\section{APPENDIX B: NUMERICAL SIMULATIONS}

For the numerical simulations, we compute the time evolution of the system according to a Lindblad master equation that accounts for experimental imperfections resulting in heating and dephasing of the motional modes [29]:

$$
\begin{aligned}
\dot{\hat{\rho}}(t)= & -i[\hat{H}, \hat{\rho}(t)] \\
& -\Gamma\left[\hat{\rho}(t) \hat{a}^{\dagger} \hat{a} \hat{a}^{\dagger} \hat{a}-2 \hat{a}^{\dagger} \hat{a} \hat{\rho}(t) \hat{a}^{\dagger} \hat{a}+\hat{a}^{\dagger} \hat{a} \hat{a}^{\dagger} \hat{a} \hat{\rho}(t)\right] \\
& -\frac{\gamma}{2} n_{\mathrm{th}}\left[\hat{a} \hat{a}^{\dagger} \hat{\rho}(t)-2 \hat{a}^{\dagger} \hat{\rho}(t) \hat{a}+\hat{\rho}(t) \hat{a} \hat{a}^{\dagger}\right] \\
& -\frac{\gamma}{2}\left(n_{\mathrm{th}}+1\right)\left[\hat{a}^{\dagger} \hat{a} \hat{\rho}(t)-2 \hat{a} \hat{\rho}(t) \hat{a}^{\dagger}+\hat{\rho}(t) \hat{a}^{\dagger} \hat{a}\right] .
\end{aligned}
$$

Here, $\Gamma$ is the dephasing parameter, which we set to be $\Gamma=(1 / \tau)$, with $\tau=2.5 \mathrm{~ms}, \gamma$ is the coupling strength between the ion motion and the thermal reservoir, and $n_{\mathrm{th}}$ is the average phonon number when the system is in equilibrium with the environment. In our model, the effective temperature of the thermal reservoir is infinite, which makes $n_{\text {th }}$ extremely large and $\gamma n_{\text {th }} \approx \gamma\left(n_{\text {th }}+1\right)$. It is natural to define the heating rate as $\gamma n_{\text {th }}$, which is measured as 70 quanta $s^{-1}$ in our system. The Hamiltonian employed in our simulations includes experimental parameters specific to our setup and takes the form of Eq. (5).

\section{APPENDIX C: PHONON-NUMBER STATE POPULATION DISTRIBUTION}

In Figs. 2 and 3, we obtain the phonon-number distribution. This is performed by driving a resonant bluesideband transition $|\downarrow, n\rangle \leftrightarrow|\uparrow, n+1\rangle$ followed by a spin projective measurement and fitting the obtained spinexcitation evolution with the formula $[11,29,35]$

$$
P_{|\uparrow\rangle}(t)=\frac{1}{2} \sum_{n} p(n)\left[1-e^{-\gamma t} \cos (\sqrt{n+1} \eta \Omega t)\right],
$$

where $p(n)$ is the phonon-number state population, $\gamma$ is an empirical decay constant, and $t$ is the pulse duration of the blue sideband. From the phonon distribution, we can then directly calculate the average phonon number that we plotted in Figs. 1(d) $-1(\mathrm{~g})$. Notice that the more populated the motional Hilbert space, the more data points are needed to fit Eq. (C1), as more $p(n)$ take part in the equation. As a consequence of this, the error bars for the expectation value of the phonon number in Figs. 1(d)-1(g) are greater for larger coupling regimes, where higher phonon numbers are involved in the dynamics.

\section{APPENDIX D: VERIFICATION OF ENTANGLEMENT FOR THE GROUND-STATE OF THE QUANTUM RABI MODEL}

In the main text, we use $\operatorname{Tr}\left[\hat{\rho}_{\text {spin }}^{2}\right]-P_{\text {rev }}^{2}<0$ to verify the existence of entanglement between the spin and the phonon degrees of freedom. This can be understood as follows. First, we introduce the purity-based entanglement witness $[56,57] \mathcal{W}$ for the target state $\hat{\rho}_{\text {tar }}$ at time $t_{\text {tar }}$, which is defined as

$$
\mathcal{W}\left[\hat{\rho}_{\text {tar }}\right] \equiv \operatorname{Tr}\left[\hat{\rho}_{\text {spin }}^{2}\right]-\operatorname{Tr}\left[\hat{\rho}_{\text {tar }}^{2}\right]
$$

It can be proven that $\mathcal{W}[\hat{\rho}] \geq 0$ for any separable state. Thus, $\mathcal{W}[\hat{\rho}]<0$ serves as a sufficient condition for the inseparability of $\hat{\rho}$. However, the purity of the whole system $\operatorname{Tr}\left[\hat{\rho}_{\mathrm{tar}}^{2}\right]$ requires the reconstruction of the total density matrix $\hat{\rho}_{\text {tar }}$ at time $t_{\text {tar }}$, which is quite demanding in our current experimental setup. Instead, after adiabatically preparing the ground state of the system, we reverse the adiabatic process, which disentangles the prepared ground state, and then measure the component $P_{\text {rev }} \equiv$ $\operatorname{Tr}\left[|\downarrow, 0\rangle\langle\downarrow, 0| \hat{\rho}_{\text {rev }}\right]$ of the spectral decomposition of the obtained final state at time $t_{\text {rev }}$, which corresponds to the probability of recovering the initial state. It is straightforward to see that $P_{\text {rev }}^{2} \leq \operatorname{Tr}\left[\hat{\rho}_{\text {rev }}^{2}\right]$. In general, unitary evolutions conserve the purity of a system, while the nonunitary contributions to the evolution that may arise during the process, mainly from random fluctuations in frequencies and intensities of lasers or the dephasing and heating of the motional modes, can only decrease the purity of the system, an effect that cannot be canceled by the reversed adiabatic process. Therefore, it is reasonable to assume that after the whole process in this experiment the purity of the system is not increased. Under such an assumption the following inequality can be established:

$$
\operatorname{Tr}\left[\hat{\rho}_{\mathrm{tar}}^{2}\right] \geq \operatorname{Tr}\left[\hat{\rho}_{\mathrm{rev}}^{2}\right] \geq P_{\mathrm{rev}}^{2}
$$

In other words, $P_{\text {rev }}^{2}$ serves as a lower bound for $\operatorname{Tr}\left[\hat{\rho}_{\text {tar }}^{2}\right]$. Putting Eqs. (D1) and (D2) together, we have

$$
\mathcal{W}\left[\hat{\rho}_{\text {tar }}\right] \leq \operatorname{Tr}\left[\hat{\rho}_{\text {spin }}^{2}\right]-P_{\text {rev }}^{2}
$$



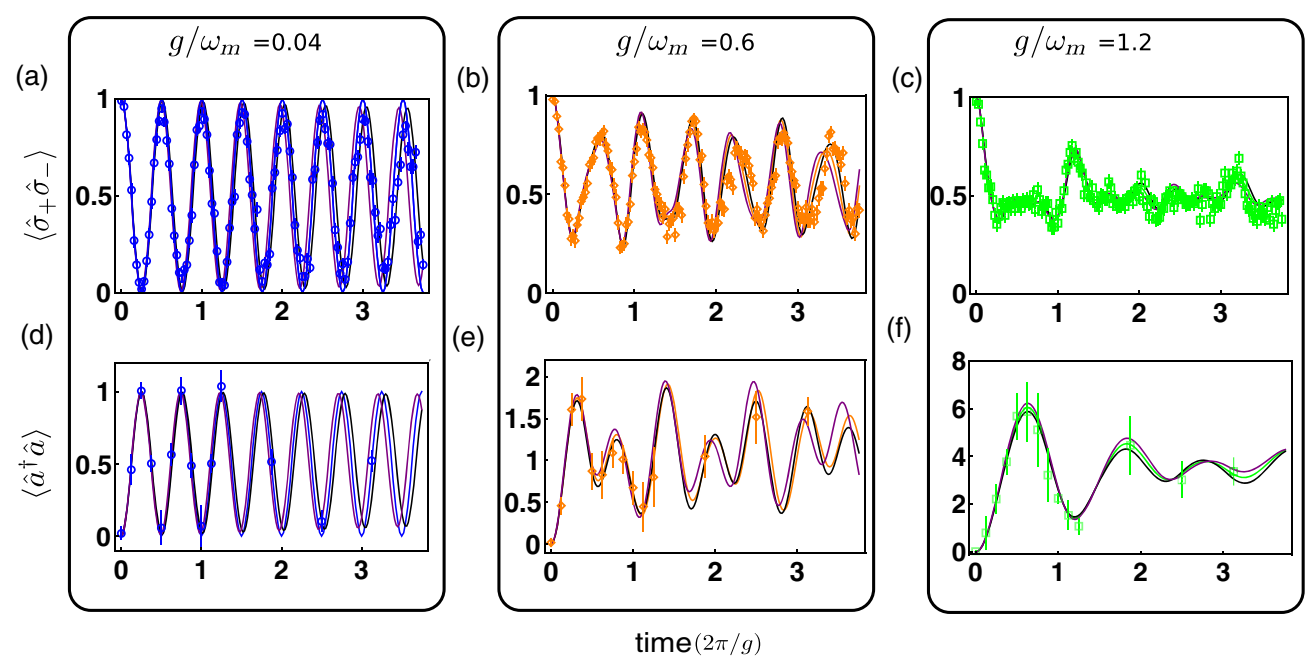

FIG. 5. Spin and phonon dynamics under the QRM for different coupling regimes with the imbalance between $\Omega_{b}$ and $\Omega_{r}$. The black and the purple curves correspond to the results of numerical simulations with $\Omega_{b}=(1-\epsilon) \Omega_{r}$ and $\Omega_{b}=(1+\epsilon) \Omega_{r}$, respectively, setting $\epsilon=3 \%$, for the cases shown Fig. 1 .

\section{APPENDIX E: CALIBRATION OF LASER INTENSITIES}

In the experiments, we calibrate the imbalance between $\Omega_{b}$ and $\Omega_{r}$ within at most $\epsilon=3 \%$, which is described by $\Omega_{b}=(1 \pm \epsilon) \Omega_{r}$. This imbalance could modify the target QRM Hamiltonian as $\hat{H}_{\mathrm{QRM}, \text { im }}$

$$
\begin{aligned}
\hat{H}_{\mathrm{QRM}, \mathrm{im}}= & \frac{\omega_{0}}{2} \hat{\sigma}_{z}+\omega_{m} \hat{a}^{\dagger} \hat{a}+g\left(1+\frac{\epsilon}{2}\right)\left(\hat{\sigma}_{+}+\hat{\sigma}_{-}\right)\left(\hat{a}+\hat{a}^{\dagger}\right) \\
& +\frac{\epsilon}{2}\left(\hat{\sigma}_{+}-\hat{\sigma}_{-}\right)\left(\hat{a}-\hat{a}^{\dagger}\right) .
\end{aligned}
$$

We observe that the effect of such an imperfection is reflected in the coupling strength $g$ that changes to $g_{\epsilon}=$ $g[1+(\epsilon /) 2]$ and in the extra term $(\epsilon / 2)\left(\hat{\sigma}_{+}-\hat{\sigma}_{-}\right)\left(\hat{a}-\hat{a}^{\dagger}\right)$. In Fig. 5, we perform numerical calculations to evaluate the effect of these terms and conclude that the main deviations from the ideal case arise from the change in coupling strength. The plotted numerical results correspond to the cases discussed in Fig. 1 with an imperfection of $\epsilon= \pm 3 \%$. No significant deviation is observed from the perfectly calibrated case.

[1] I. I. Rabi, On the Process of Space Quantization, Phys. Rev. 49, 324 (1936).

[2] R. Frisch and E. Segre, Über die Einstellung der Richtungsquantelung. II, Z. Phys. 80, 610 (1933).

[3] E. T. Jaynes and F. W. Cummings, Comparison of Quantum and Semiclassical Radiation Theories with Application to the Beam Maser, Proc. IEEE 51, 89 (1963).

[4] R. Miller, T. E. Northup, K. M. Birnbaum, A. Boca, A. D. Boozer, and H. J. Kimble, Trapped Atoms in Cavity QED:
Coupling Quantized Light and Matter, J. Phys. B 38, S551 (2005).

[5] H. Walther, B. T. H. Varcoe, B. G. Englert, and T. Becker, Cavity Quantum Electrodynamics, Rep. Prog. Phys. 69, 1325 (2006).

[6] J.-M. Raimond and S. Haroche, Exploring the Quantum: Atoms, Cavities, and Photons (Oxford University Press, Oxford, 2006), pp. 151-161.

[7] H. Ritsch, P. Domokos, F. Brennecke, and T. Esslinger, Cold Atoms in Cavity-Generated Dynamical Optical Potentials, Rev. Mod. Phys. 85, 553 (2013).

[8] R. Hanson, L. P. Kouwenhoven, J. R. Petta, S. Tarucha, and L. M. K. Vandersypen, Spins in Few-Electron Quantum Dots, Rev. Mod. Phys. 79, 1217 (2007).

[9] A. Wallraff, D. I. Schuster, A. Blais, L. Frunzio, R.-S. Huang, J. Majer, S. Kumar, S. M. Girvin, and R. J. Schoelkopf, Strong Coupling of a Single Photon to a Superconducting Qubit Using Circuit Quantum Electrodynamics, Nature (London) 431, 162 (2004).

[10] M. H. Devoret and R. J. Schoelkopf, Superconducting Circuits for Quantum Information: An Outlook, Science 339, 1169 (2013).

[11] D. M. Meekhof, C. Monroe, B. E. King, W. M. Itano, and D. J. Wineland, Generation of Nonclassical Motional States of a Trapped Atom, Phys. Rev. Lett. 76, 1796 (1996).

[12] D. Leibfried, R. Blatt, C. Monroe, and D. J. Wineland, Quantum Dynamics of Single Trapped Ions, Rev. Mod. Phys. 75, 281 (2003).

[13] H. Häffner, C. F. Roos, and R. Blatt,, Quantum Computing with Trapped Ions, Phys. Rep. 469, 155 (2008).

[14] D. Lv, S. An, M. Um, J. Zhang, J.-N. Zhang, M. S. Kim, and K. Kim, Reconstruction of the Jaynes-Cummings Field State of Ionic Motion in a Harmonic Trap, Phys. Rev. A 95, 043813 (2017).

[15] T. Niemczyk, F. Deppe, H. Huebl, E. P. Menzel, F. Hocke, M. J. Schwarz, J. J. Garcia-Ripoll, D. Zueco, T. Hümmer, E. Solano, A. Marx, and R. Gross, Circuit Quantum 
Electrodynamics in the Ultrastrong-Coupling Regime, Nat. Phys. 6, 772 (2010).

[16] P. Forn-Díaz, J. J. García-Ripoll, B. Peropadre, J.-L. Orgiazzi, M. A. Yurtalan, R. Belyansky, C. M. Wilson, and A. Lupascu, Ultrastrong Coupling of a Single Artificial Atom to an Electromagnetic Continuum in the Nonperturbative Regime, Nat. Phys. 13, 39 (2017).

[17] F. Yoshihara, T. Fuse, S. Ashhab, K. Kakuyanagi, S. Saito, and K. Semba, Superconducting Qubit-Oscillator Circuit beyond the Ultrastrong-Coupling Regime, Nat. Phys. 13, 44 (2017).

[18] D. Z. Rossatto, C. J. Villas-Bôas, M. Sanz, and E. Solano, Spectral Classification of Coupling Regimes in the Quantum Rabi Model, Phys. Rev. A 96, 013849 (2017).

[19] J. Casanova, G. Romero, I. Lizuain, J. J. García-Ripoll, and E. Solano, Deep Strong Coupling Regime of the JaynesCummings Model, Phys. Rev. Lett. 105, 263603 (2010).

[20] G. Romero, D. Ballester, Y. M. Wang, V. Scarani, and E. Solano, Ultrafast Quantum Gates in Circuit QED, Phys. Rev. Lett. 108, 120501 (2012).

[21] D. Braak, Integrability of the Rabi Model, Phys. Rev. Lett. 107, 100401 (2011).

[22] J. S. Pedernales, I. Lizuain, S. Felicetti, G. Romero, L. Lamata, and E. Solano, Quantum Rabi Model with Trapped Ions, Sci. Rep. 5, 15472 (2015).

[23] J. Lolli, A. Baksic, D. Nagy, V. E. Manucharyan, and C. Ciuti, Ancillary Qubit Spectroscopy of Vacua in Cavity and Circuit Quantum Electrodynamics, Phys. Rev. Lett. 114, 183601 (2015).

[24] T. Jaako, Z.-L. Xiang, J. J. Garcia-Ripoll, and P. Rabl, Ultrastrong-Coupling Phenomena beyond the Dicke Model, Phys. Rev. A 94, 033850 (2016).

[25] C. K. Andersen and A. Blais, Ultrastrong Coupling Dynamics with a Transmon Qubit, New J. Phys. 19, 023022 (2017).

[26] A. Crespi, S. Longhi, and R. Osellame, Photonic Realization of the Quantum Rabi Model, Phys. Rev. Lett. 108, 163601 (2012).

[27] J. Braumüller, M. Marthaler, A. Schneider, A. Stehli, H. Rotzinger, M. Weides, and A. V. Ustinov, Analog Quantum Simulation of the Rabi Model in the Ultra-Strong Coupling Regime, Nat. Commun. 8, 779 (2017).

[28] N. K. Langford, R. Sagastizabal, M. Kounalakis, C. Dickel, A. Bruno, F. Luthi, D. J. Thoen, A. Endo, and L. DiCarlo, Experimentally Simulating the Dynamics of Quantum Light and Matter at Deep-Strong Coupling, Nat. Commun. 8, 1715 (2017).

[29] S.-M. An, J.-N. Zhang, M. Um, D.-S. Lv, Y. Lu, J.-H. Zhang, Z.-Q. Yin, H.-T. Quan, and K. Kim, Experimental Test of the Quantum Jarzynski Equality with a Trapped-Ion System, Nat. Phys. 11, 193 (2015).

[30] C. Monroe, D. M. Meekhof, B. E. King, S. R. Jefferts, W. M. Itano, D. J. Wineland, and P. Gould, ResolvedSideband Raman Cooling of a Bound Atom to the 3D Zero-Point Energy, Phys. Rev. Lett. 75, 4011 (1995).

[31] C. Monroe, D. M. Meekhof, B. E. King, and D. J. Wineland, A Schrödinger Cat Superposition State of an Atom, Science 272, 1131 (1996).

[32] P. C. Haljan, K. A. Brickman, L. Deslauriers, P. J. Lee, and C. Monroe, Spin-Dependent Forces on Trapped Ions for
Phase-Stable Quantum Gates and Entangled States of Spin and Motion, Phys. Rev. Lett. 94, 153602 (2005).

[33] M. J. McDonnell, J. P. Home, D. M. Lucas, G. Imreh, B. C. Keitch, D. J. Szwer, N. R. Thomas, S. C. Webster, D. N. Stacey, and A. M. Steane, Long-Lived Mesoscopic Entanglement Outside the Lamb-Dicke Regime, Phys. Rev. Lett. 98, 063603 (2007).

[34] U. Poschinger, A. Walther, K. Singer, and F. Schmidt-Kaler, Observing the Phase Space Trajectory of an Entangled Matter Wave Packet, Phys. Rev. Lett. 105, 263602 (2010).

[35] H-Y. Lo, D. Kienzler, L. de Clercq, M. Marinelli, V. Negnevitsky, B. C. Keitch, and J. P. Home, Spin-Motion Entanglement and State Diagnosis with Squeezed Oscillator Wavepackets, Nature (London) 521, 336 (2015).

[36] S.-Q. Ding, G. Maslennikov, R. Hablützel, H. Loh, and D. Matsukevich, Quantum Parametric Oscillator with Trapped Ions, Phys. Rev. Lett. 119, 150404 (2017).

[37] A. Sørensen and K. Mølmer, Quantum Computation with Ions in Thermal Motion, Phys. Rev. Lett. 82, 1971 (1999).

[38] A. Sø rensen and K. Mø lmer, Entanglement and Quantum Computation with Ions in Thermal Motion, Phys. Rev. A 62 , 022311 (2000).

[39] C. A. Sackett, D. Kielpinski, B. E. King, C. Langer, V. Meyer, C. J Myatt, M. Rowe, Q. A. Turchette, W. M. Itano, D. J. Wineland, and C. Monroe, Experimental Entanglement of Four Particles, Nature (London) 404, 256 (2000).

[40] D. G. Leibfried, B. L. DeMarco, V. Meyer, D. Lucas, M. D. Barrett, J. W. Britton, W. M. Itano, B. M. Jelenković, C. Langer, T. P. Rosenband, and D. J. Wineland, Experimental Demonstration of a Robust, High-Fidelity Geometric Two Ion-Qubit Phase Gate, Nature (London) 422, 412 (2003).

[41] J. Benhelm, G. Kirchmair, C. F. Roos, and R. Blatt, Towards Fault-Tolerant Quantum Computing with Trapped Ions, Nat. Phys. 4, 463 (2008).

[42] D. Hayes, D. N. Matsukevich, P. Maunz, D. Hucul, Q. Quraishi, S. Olmschenk, W. Campbell, J. Mizrahi, C. Senko, and C. Monroe, Entanglement of Atomic Qubits Using an Optical Frequency Comb, Phys. Rev. Lett. 104, 140501 (2010).

[43] L. Lamata, J. León, T. Schätz, and E. Solano, Dirac Equation and Quantum Relativistic Effects in a Single Trapped Ion, Phys. Rev. Lett. 98, 253005 (2007).

[44] R. Gerritsma, G. Kirchmair, F. Zähringer, E. Solano, R. Blatt, and C. F. Roos, Quantum Simulation of the Dirac Equation, Nature (London) 463, 68 (2010).

[45] X. Zhang, Y.-C. Shen, J.-H. Zhang, J. Casanova, L. Lamata, E. Solano, M. H. Yung, J.-N. Zhang, and K. Kim, Time Reversal and Charge Conjugation in an Embedding Quantum Simulator, Nat. Commun., 6, 7971 (2015).

[46] M. A. Nielson and I. L. Chuang, Quantum Computation and Quantum Information (Cambridge University Press, Cambridge, England, 2000), pp. 98-108, 409-414.

[47] C. F. Roos, G. P. T. Lancaster, M. Riebe, H. Häffner, W. Hänsel, S. Gulde, C. Becher, J. Eschner, F. Schmidt-Kaler, and R. Blatt, Bell States of Atoms with Ultralong Lifetimes and Their Tomographic State Analysis, Phys. Rev. Lett. 92, 220402 (2004). 
[48] C. Senko, J. Smith, P. Richerme, A. Lee, W. C. Campbell, and C. Monroe, Coherent Imaging Spectroscopy of a Quantum Many-Body Spin System, Science 345, 430 (2014).

[49] M. J. Hwang, R. Puebla, and M. B. Plenio,, Quantum Phase Transition and Universal Dynamics in the Rabi Model, Phys. Rev. Lett. 115, 180404 (2015).

[50] R. Puebla, M.-J. Hwang, J. Casanova, and M. B. Plenio, Probing the Dynamics of a Superradiant Quantum Phase Transition with a Single Trapped Ion, Phys. Rev. Lett. 118, 073001 (2017).

[51] M. J. Hwang, P. Rabl, and M. B. Plenio, Dissipative Phase Transition in the Open Quantum Rabi Model, Phys. Rev. A 97, 013825 (2018).

[52] R.H. Dicke, Coherence in Spontaneous Radiation Processes, Phys. Rev. 93, 99 (1954).
[53] V. M. Bastidas, C. Emary, B. Regler, and T. Brandes, Nonequilibrium Quantum Phase Transitions in the Dicke Model, Phys. Rev. Lett. 108, 043003 (2012).

[54] L. Bakemeier, A. Alvermann, and H. Fehske, Quantum Phase Transition in the Dicke Model with Critical and Noncritical Entanglement, Phys. Rev. A 85, 043821 (2012).

[55] P. J. Lee, K. A. Brickman, L. Deslauriers, P. C. Haljan, L.-M. Duan, and C. Monroe, Phase Control of Trapped Ion Quantum Gates, J. Opt. B 7, S371 (2005).

[56] R. Islam, R. Ma, P. M. Preiss, M. E. Tai, A. Lukin, M. Rispoli, and M. Greiner, Measuring Entanglement Entropy in a Quantum Many-Body System, Nature (London) 528, 77 (2015).

[57] R. Horodecki, P. Horodecki, M. Horodecki, and K. Horodecki, Quantum Entanglement, Rev. Mod. Phys. 81, 865 (2009). 